\title{
On the Galois groups of generalized Laguerre Polynomials
}

\author{
Shanta Laishram
}

Abstract. For a positive integer $n$ and a real number $\alpha$, the generalized Laguerre polynomials are defined by

$$
L_{n}^{(\alpha)}(x)=\sum_{j=0}^{n} \frac{(n+\alpha)(n-1+\alpha) \cdots(j+1+\alpha)(-x)^{j}}{j !(n-j) !} .
$$

These orthogonal polynomials are solutions to Laguerre's Differential Equation which arises in the treatment of the harmonic oscillator in quantum mechanics. Schur studied these Laguerre polynomials for their interesting algebraic properties. In this short article, it is shown that the Galois groups of Laguerre polynomials $L_{n}^{(\alpha)}(x)$ is $S_{n}$ with $\alpha \in\left\{ \pm \frac{1}{2}, \pm \frac{1}{3}, \pm \frac{2}{3}, \pm \frac{1}{4}, \pm \frac{3}{4}\right\}$ except when $(\alpha, n) \in\left\{\left(\frac{1}{4}, 2\right),\left(-\frac{2}{3}, 11\right),\left(\frac{2}{3}, 7\right)\right\}$. The proof is based on ideas of $p$-adic Newton polygons.

Keywords. Irreducibility, Laguerre Polynomials, Newton Polygons, Arithmetic Progressions, Primes.

2010 Mathematics Subject Classification. 2000 Mathematics Subject Classification: Primary 11A41, 11B25, 11N05, 11N13, $11 \mathrm{C} 08,11 \mathrm{Z} 05$

\section{Introduction}

For a positive integer $n$ and a real number $\alpha$, the generalized Laguerre polynomials are defined by

$$
L_{n}^{(\alpha)}(x)=\sum_{j=0}^{n} \frac{(n+\alpha)(n-1+\alpha) \cdots(j+1+\alpha)(-x)^{j}}{j !(n-j) !} .
$$

These orthogonal polynomials have a wide range of applications in several areas of mathematics. Not long after its appearance in the literature early in the twentieth century, it became evident, in the hands of Schur, that the generalized Laguerre polynomials also enjoy algebraic properties of great interest. In fact the irreducibility of these polynomials is connected to finding explicit examples of solutions to Hilbert's Inverse Galois Problem. We refer to [FKT12] for more details.

It was shown that $L^{(\alpha)}(x)$ is irreducible for $\alpha \in\left\{ \pm \frac{1}{2}\right\}$ in [Sch29] and [Sch31] and for $\alpha \in$ $\left\{ \pm \frac{1}{3}, \pm \frac{2}{3}, \pm \frac{1}{4}, \pm \frac{3}{4}\right\}$ in [LaSh, Theorem 1] except when $\alpha=\frac{1}{4}, n=2$. By using these results of irreducibility, it was shown in [SaSh15, Theorem 1.4] that the Galois group of $L^{(\alpha)}(x)$ is $S_{n}$ for $n \geq n_{0}$ where $n_{0}=182,876,1325$ if $q \in\left\{ \pm \frac{1}{2}\right\}, q \in\left\{ \pm \frac{1}{3}, \pm \frac{2}{3}\right\}$ and $q \in\left\{ \pm \frac{1}{4}, \pm \frac{3}{4}\right\}$, respectively. In this short note, we give a complete result for all $n$. Here $S_{n}$ is the Symmetric Group on $n$ symbols and $A_{n}$ is the Alternating Group on $n$ symbols. We prove

Theorem 1.1. Let $\alpha \in\left\{ \pm \frac{1}{2}, \pm \frac{1}{3}, \pm \frac{2}{3}, \pm \frac{1}{4}, \pm \frac{3}{4}\right\}$. The Galois group of Laguerre polynomials $L_{n}^{(\alpha)}(x)$ is $S_{n}$ for every $n \geq 1$ except when $(\alpha, n) \in\left\{\left(\frac{1}{4}, 2\right),\left(-\frac{2}{3}, 11\right),\left(\frac{2}{3}, 7\right)\right\}$ where it is $A_{n}$ for $(\alpha, n) \in$ $\left\{\left(-\frac{2}{3}, 11\right),\left(\frac{2}{3}, 7\right)\right\}$ and $S_{1}$ for $(\alpha, n)=\left(\frac{1}{4}, 2\right)$.

We give a proof of Theorem 1.1 in Section 3. The proof of Theorem 1.1 is an application of a result of Hajir [Haj05] based on $p$-adic Newton polgons, see Lemmas 2.1 and 2.2. The new ingredient in this paper is the clever use of Lemma 2.1 as Lemma 2.2 instead of [SaSh15, Lemma 3.3]. In fact the proof of [SaSh15, Theorem 1.4] can be much shortened by using Lemma 2.2. 


\section{Preliminaries}

Hajir [Haj05] gave a criterion for an irreducible polynomial to have large Galois group using Newton polygons. We restate the result which is [Haj05, Lemma 3.1].

Lemma 2.1. Let $f(x)=\sum_{j=0}^{m}\left(\begin{array}{c}m \\ j\end{array}\right) c_{j} x^{j} \in \mathbb{Q}[X]$ be an irreducible polynomial of degree $m$. Let $p$ be a prime with $\frac{m}{2}<p<m-2$ such that

(i) $\operatorname{ord}_{p}\left(c_{0}\right)=1$,

(ii) $\operatorname{ord}_{p}\left(c_{j}\right) \geq 1$ for $0 \leq j \leq m-p$,

(iii) $\operatorname{ord}_{p}\left(c_{p}\right)=0$.

Then $p$ divides the order of Galois group of $f$ over $\mathbb{Q}$. In fact, this Galois group is $A_{m}$ if $\operatorname{disc}(f) \in \mathbb{Q}^{* 2}$ and $S_{m}$ otherwise.

We will be applying the above lemma to following polynomial. Let $\alpha=\frac{u}{v}$ with $u, v \in \mathbb{Z}, \operatorname{gcd}(u, v)=$ 1 and $v>0$. Let

$$
\begin{aligned}
G(x, u, v): & =v^{n} n ! L^{\left(\frac{u}{v}\right)}\left(\frac{-x}{v}\right) \\
& =\sum_{j=0}^{n}\left(\begin{array}{l}
n \\
j
\end{array}\right)(u+v n)(u+v(n-1)) \cdots(u+v(j+1)) x^{j} .
\end{aligned}
$$

In [Sch31], Schur showed that its discriminant is given by

$$
D_{n}^{(u, v)}:=\operatorname{Disc}(G(x, u, v))=\prod_{j=2}^{n} j^{j}\left(\frac{u}{v}+j\right)^{j-1} .
$$

We write $D_{m}^{(u, v)}=b Y^{2}, Y \in \mathbb{Q}$ with

$$
b= \begin{cases}\frac{3 \cdot 5 \cdots n \cdot(u+2 v)(u+4 v) \cdots(u+(n-1) v)}{v^{\delta}} & \text { if } n \equiv 1,3(\bmod 4) \\ \frac{3 \cdot 5 \cdots(n-1) \cdot(u+2 v)(u+4 v) \cdots(u+n v)}{v^{\delta}} & \text { if } n \equiv 0,2(\bmod 4)\end{cases}
$$

where $\delta=0$ if $n \equiv 0,1(\bmod 4)$ and 1 if $n \equiv 2,3(\bmod 4)$.

We now apply Lemma 2.1 to $G(x, u, v)$. We prove

Lemma 2.2. Let $1 \leq r<v, \operatorname{gcd}(r, v)=1$ and $p$ be a prime with

$$
p>v, p \equiv r^{-1} u(\bmod v) \text { and } \frac{u+v+n v}{r+v} \leq p \leq n-3 .
$$

Let $G(x, u, v)$ be given by (2.1) be an irreducible polynomial of degree $n$. Assume that $|u|<v$. Then the Galois group of $G(x, u, v)$ is $A_{n}$ or $S_{n}$ according as b (given by (2.2)) is a square or not an square of an integer.

Proof. We apply Lemma 2.1 with

$$
c_{j}=(u+v n)(u+v(n-1)) \cdots(u+v(j+1)) .
$$

Since $1 \leq r<v$, we have $\frac{u+v+n v}{r+v}>\frac{n}{2}$ and hence $\frac{n}{2}<p<n-2$ is valid. It suffices to check conditions (i) - (iii) of Lemma 2.1. 
Since $p \equiv r^{-1} u(\bmod v)$, we get $p \mid(u+i v)$ for some $i$. Let $i_{0}$ be the least positive integer $i$ with this property. Then $1 \leq i_{0}<p$. Further let $u+i_{0} v=p r_{0}$. Then $r_{0} \equiv r(\bmod v)$. We claim that $r_{0}<v$. Suppose not. Then $u+i_{0} v=p r_{0} \geq p v \geq\left(i_{0}+1\right) v$ since $i_{0}<p$ contradicting $|u|<v$. Thus $r_{0}<v$. This with $r_{0} \equiv r(\bmod v)$ and $1 \leq r<v$ implies $r=r_{0}$. Since $\frac{u+v+n v}{r+v} \leq p$, we have

$$
u+v+(n-p) v \leq r p=r_{0} p=u+i_{0} v
$$

giving $i_{0}>n-p$. Thus $n-p<i_{0}<p$. This gives $i_{0}-p<0$ and $i_{0}+p>n$ and hence $u+i_{0} v$ is the only multiple of $p$ in $\{u, u+v, \cdots, u+n v\}$. Further $u+i_{0} v=p r<p v<p^{2}$ implying $p \|\left(u+i_{0} v\right)$. Hence conditions $(i)-($ iii $)$ of Lemma 2.1 are valid and the assertion follows.

The above Lemma contains [SaSh15, Lemma 3.3]. We also need the following result on $b$ being a square or not.

Lemma 2.3. Let $2 \leq n \leq 1325$,

$$
\alpha=\frac{u}{v} \in\left\{ \pm \frac{1}{2}, \pm \frac{1}{3}, \pm \frac{2}{3}, \pm \frac{1}{4}, \pm \frac{3}{4}\right\}
$$

and $b$ be given by (2.2). Then $b$ is square only when $(u, v, n) \in\{(-2,3,3),(-2,3,11),(2,3,7)\}$.

Proof. First we check that for $2 \leq n \leq u+2 v$, the assertion is valid. Hence we now take $n>u+2 v$. Let

$$
n_{1}= \begin{cases}\frac{n}{2} & \text { if } n \text { is even } \\ \frac{n-1}{2} & \text { if } n \text { is odd }\end{cases}
$$

Assume $u \neq \pm 2$ if $v=3$. Then we see that $b$ is divisible by every prime $p \equiv u(\bmod 2 v)$ with $n<p \leq u+2 v n_{1}$ to the first power. Hence if there is such a prime, $b$ cannot be a square. For $u+2 v<n \leq 1325$, we check that this is true. Thus we now take $v=3, u= \pm 2$. Let $u_{1}=\frac{u}{2}$. Then

$$
(u+2 v) \cdots\left(u+2 n_{1} v\right)=2^{n_{1}}\left(u_{1}+v\right)\left(u_{1}+2 v\right) \cdots\left(u_{1}+n_{1} v\right)
$$

and hence $b$ is not an square if there is a prime $p$ with $n<p \leq u_{1}+n_{1} v$ and $p \equiv u_{1}(\bmod v)$ where $v=3$. We check that this is the case for $u+2 v<m \leq 1325$ except when $u=-2, m \in\{5,6,7,11\}$ and $u=2, m=19$. For $u=-2, m \in\{5,6,7,11\}$ and $u=2, m=19$, we check that $b$ is not a square except when $u=-2, m=11$. Hence the assertion follows.

\section{Proof of Theorem 1.1}

Let

$$
\alpha=\frac{u}{v} \in\left\{ \pm \frac{1}{2}, \pm \frac{1}{3}, \pm \frac{2}{3}, \pm \frac{1}{4}, \pm \frac{3}{4}\right\}
$$

As mentioned before, it was shown that $L^{(\alpha)}(x)$ is irreducible for $\alpha \in\left\{ \pm \frac{1}{2}\right\}$ in [Sch29] and [Sch31] and in [LaSh] for $\alpha \in\left\{ \pm \frac{1}{3}, \pm \frac{2}{3}, \pm \frac{1}{4}, \pm \frac{3}{4}\right\}$ except when $\alpha=\frac{1}{4}, n=2$ and hence same is true for $G(x, u, v)$. For $n \leq 13$, we check in $S A G E$ for $n \leq 11$ and MAGMA for $n=12,13$ that the assertion of Theorem 1.1 is valid.

Hence we may suppose that $n>13$. Further we can take $n \leq 1325$ by [SaSh15, Theorem 1.4]. It suffices to prove that $G(x, u, v)$ has Galois group $S_{n}$. We use Lemmas 2.2 and 2.2. It suffices to find a prime $p$ with

$$
p>v, p \equiv r^{-1} u(\bmod v) \text { and } \frac{u+v+n v}{r+v} \leq p \leq n-3 .
$$


for some $r, 1 \leq r<v, \operatorname{gcd}(r, v)=1$. Let $\alpha=\frac{u}{v} \in\left\{ \pm \frac{1}{2}\right\}$. We check that there is a prime $p$ with $\frac{2 n+2+u}{3} \leq p \leq n-3$ except when $u=1, n=19$. We check that for $n=19$, the Galois group of $L^{\left(\frac{1}{2}\right)}(x)$ is $S_{n}$.

Let $\alpha=\frac{u}{v} \in\left\{ \pm \frac{1}{3}, \pm \frac{2}{3}\right\}$. Since $1 \leq r<3$, we need to find a prime $p$ with

$$
\frac{3 n}{4}+\frac{3+u}{4} \leq p \leq n-3, p \equiv u(\bmod 3)
$$

or

$$
\frac{3 n}{5}+\frac{3+u}{5} \leq p \leq n-3, p \equiv 2 u(\bmod 3) .
$$

Hence it suffices to find a prime $p$ with $\frac{3 n}{4}+\frac{3+u}{4} \leq p \leq n-3$ or

$$
\frac{3 n}{5}+\frac{3+u}{5} \leq p<\frac{3 n}{4}+\frac{3+u}{4}, p \equiv 2 u(\bmod 4) .
$$

We check that this is the case except when

$$
\begin{aligned}
& u=-1: n=15 \\
& u=-2: n \in=19 \\
& u=1: n \in\{18,19\} \\
& u=2: n \in\{14,15,31\} .
\end{aligned}
$$

For these values of $\frac{u}{3}$ and $n$, we check in MAGMA that Galois group of $L^{\left(\frac{u}{3}\right)}(x)$ is $S_{n}$.

Let $\alpha=\frac{u}{v} \in\left\{ \pm \frac{1}{4}, \pm \frac{3}{4}\right\}$. Since $r \in\{1,3\}$, we need to find a prime $p$ with

$$
\frac{4 n}{5}+\frac{4+u}{5} \leq p \leq n-3, p \equiv u(\bmod 4)
$$

or

$$
\frac{4 n}{7}+\frac{4+u}{7} \leq p \leq n-3, p \equiv 3 u(\bmod 4) .
$$

Hence it suffices to find a prime $p$ with $\frac{4 n}{5}+\frac{4+u}{5} \leq p \leq n-3$ or

$$
\frac{4 n}{7}+\frac{4+u}{7} \leq p<\frac{4 n}{5}+\frac{4+u}{5}, p \equiv 3 u(\bmod 4)
$$

We check that this is the case except when

$$
\begin{aligned}
& u=-1: n \in\{14,15,30,31\} \\
& u=-3: n \in\{20,21,23\} \\
& u=1: n=19 \\
& u=3: n \in\{14,29,30,31\} .
\end{aligned}
$$

For these values of $\frac{u}{4}$ and $n$, we check in MAGMA that Galois group of $L^{\left(\frac{u}{4}\right)}(x)$ is $S_{n}$. This completes the proof of Theorem 1.1.

\section{Acknoweldgements}

We thank the anonymous referee for the remarks in an earlier version of this paper. We also thank DST for supporting this work under the Fast Track Project. 


\section{References}

[FKT12] M. Filaseta, T. Kidd and O. Trifonov, Laguerre polynomials with Galois group $A_{m}$ for each $m$, J. Number Theory, 132 (2012), no. 4, 776-805.

[Haj05] F. Hajir, On the Galois group of generalized Laguerre polynomials, J. Théor. Nombres Bordeaux, 17(2) (2005), $517-525$.

[LaSh] S. Laishram and T. N. Shorey, Irreducibility of generalized Hermite-Laguerre polynomials III, Submitted.

[SaSh15] N. Saradha and T. N. Shorey, Squares in blocks from an arithmetic progression and Galois group of Laguerre polynomials, Int. Jour. of Number Theory, 11(1) (2015), 233-250.

[Sch29] I. Schur, Einige Sätze über Primzahlen mit Anwendungen auf Irreduzibilitätsfragen, II, Sitzungsber. Preuss. Akad. Wiss. Berlin Phys.-Math. Kl., 14 (1929), 370-391.

[Sch31] I. Schur, Affektlose Gleichungen in der Theorie der Laguerreschen und Hermitschen Polynome, J. Reine Angew. Math., 165 (1931), 52-58.

\section{Shanta Laishram}

Stat-Math Unit, Indian Statistical Institute

7 S. J. S. Sansanwal Marg

New Delhi, 110016, India.

e-mail: shanta@isid.ac.in 